\section{Bacterial blood brain bypass}

\section{By Chris Cain, Staff Writer}

A team at Institut National de la Santé et de la Recherche Médicale has identified a new mechanism that explains how meningococcus crosses the blood brain barrier. The researchers think the findings could be used to treat one form of meningococcal sepsis and may also have broader applications in delivering therapeutics into the CNS.

Neisseria meningitidis (meningococcus) is a bacterial pathogen that causes disease in about 1 in 100,000 people in the U.S. each year, primarily in children less than 1 year old. Although the disease is both rare and readily prevented with vaccines, researchers at the Institut National de la Santé et de la Recherche Médicale (INSERM) have maintained interest in the bacteria's rare ability to bypass the blood brain barrier (BBB) and induce swelling of the brain.

In 2009, an INSERM group published in Science that meningococcus bypasses the BBB by creating gaps in the normally impenetrable tight junctions that form the barrier's cell-cell interface. ${ }^{1}$

Now, the team has fleshed out the precise mechanism² (see Figure 1, "Breaching the blood brain barrier"). First, type IV pili, which are thread-like protein structures expressed on the bacterial surface, bind to adrenergic receptor $\beta_{2}$ (ADRB2) expressed on the surface of cells lining the BBB. An ADRB2-associated scaffolding protein, arrestin $\beta 2$ (ARRB2), then signals downstream to trigger rearrangement of the host cell cytoskeleton, stopping meningococcus from being washed away by the shear stress of blood flow against the barrier (see Figure 1[a]).

Next, ARRB2 recruits the tight junction proteins catenin (cadherin-associated protein) $\delta 1$ (CTNND1; p120) and VE-cadherin (CD144; cadherin-5) away from the cell-cell interface to the site of bacterial adhesion (see Figure $\mathbf{1}[\mathbf{b}]$ ). This weakens the tight junction and creates gaps in the $\mathrm{BBB}$, which meningococcus can then invade (see Figure 1 $[\mathbf{c}]$ ).

The researchers also showed that the generic adrenergic receptor $\beta$ agonist isoproterenol prevented meningococcal invasion in a cell culture model of the BBB.

The findings were published in Cell.

Stefano Marullo, a research director at INSERM and senior author of the Cell paper, said the work may have therapeutic implications for some meningococcal-infected patients who experience sepsis.

"In a subset of patients you see the formation of red spots on the skin, known as purpura fulminans, which indicates that bacteria are causing the leakage of blood from the capillaries. This can kill a completely healthy person within four hours. Treatment with an adrenergic receptor $\beta$ agonist would cause internalization of the receptor away from the cell surface and could rapidly treat these patients," said Marullo.

He did say more work needs to be done to see if the drug affects peripheral capillaries in the same way it affects the distinct, tightly-knit capillaries that line the BBB. At least 15 companies have adrenergic receptor $\beta$ agonists in development or marketed to treat asthma and chronic obstructive pulmonary disease (COPD).

Xavier Nassif, a research director at INSERM, was a senior author on both publications and filed a patent covering the use of meningococcal pilin proteins to deliver therapeutics across the BBB.

"I could envision using a short, pilin-derived peptide of 20-30 amino acids to enable delivery of drugs, perhaps carried in liposomes, across the blood brain barrier," he told SciBX.

Nassif said additional experiments need to be done, including testing purified pilin protein in cell culture and eventually in mouse models.

"One important experiment would be to show the offset of the effect: How long is the barrier open after giving pilin proteins? Ideally, to deliver a drug into the brain one would like to open the barrier long enough so that a drug can get in, but this should also be as short as possible to minimize the time during which plasma proteins or other compounds could get in the brain and cause damage," said Bjoern Bauer, assistant professor in the College of Pharmacy at the University of Minnesota.

He also was concerned that purified protein may not specifically target the $\mathrm{BBB}$, as many cells express ADRB2. This could cause side effects and would make specific delivery to the brain difficult, according to Bauer.

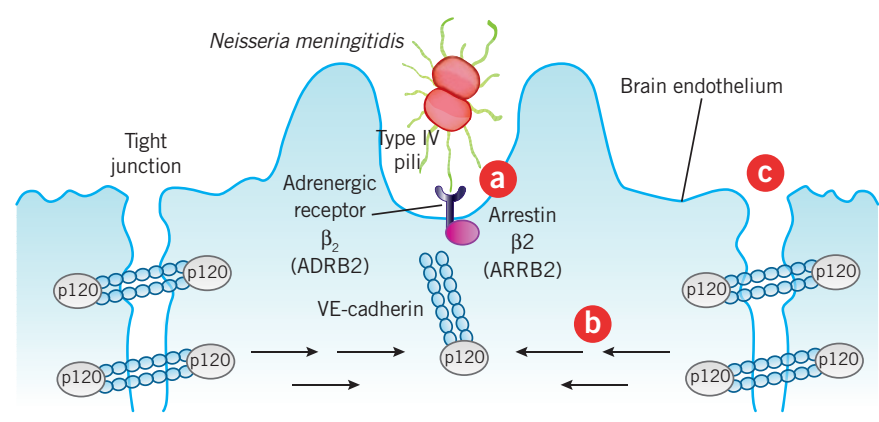

Figure 1. Breaching the blood brain barrier. 


\section{ANALYSIS}

TARGETS \& MECHANISMS

Bauer leads a research team focused on delivering drugs across the $\mathrm{BBB}$. The group's strategy is to transiently inhibit drug efflux transporters, which would allow a window of time to deliver drugs into the CNS.

Nassif and Marullo now plan to map the pili-interacting region of ADRB2 and in the future intend to further explore the signaling pathway triggered by pili binding to the receptor.

Cain, C. SciBX 4(3); doi:10.1038/scibx.2011.64

Published online Jan. 20, 2011
REFERENCES

1. Coureuil, M. et al. Science 325, 83-87 (2009)

2. Coureuil, M. et al. Cell; published online Dec. 23, 2010; doi:10.1016/j.cell.2010.11.035

Contact: Stefano Marullo, University Paris Descartes and Cochin Institute, Paris, France e-mail: stefano.marullo@inserm.fr

COMPANIES AND INSTITUTIONS MENTIONED Institut National de la Santé et de la Recherche Médicale, Paris, France

University of Minnesota, Duluth, Minn. 